\title{
STUDY OF THE EFFECTS OF AMBIENT CONDITIONS UPON THE PERFORMANCE OF FAN POWERED, INFRARED, NATURAL GAS BURNERS$$
\text { DOE/MT/ } 94011--8
$$ \\ Quarterly Technical Progress Report

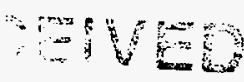 \\ $\operatorname{sip} 101990$ \\ For the Period April 01, 1996 -- June 30, 1996 \\ OSTI
}

Tiejun Bai (PI), Yaw D. Yeboah, and Ramanathan Sampath

July 1996

Grant No. DE-FG22-94MT94011 \&

For

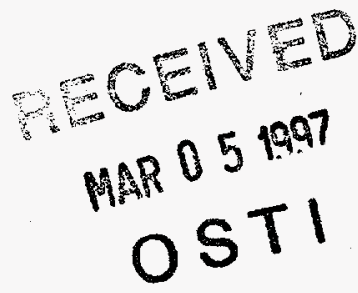

U.S. Department of Energy

Pittsburgh Energy Technology Center

Attn: Document Control Center

P.O. Box 10940, MS 921-143

Pittsburgh, PA 15236-0940

By

Department of Engineering

Clark Atlanta University

Atlanta, GA 30314

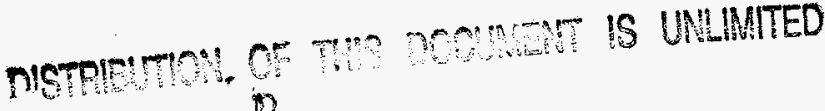

US/DOE PATENT CLEARANCE IS NOT REQUIRED PRIOR TO THE PUBLICATION OF THIS DOCUMENT 


\section{DISCLAMMER}

Portions of this document may be illegible in electronic image products. Images are produced from the best available original document. 


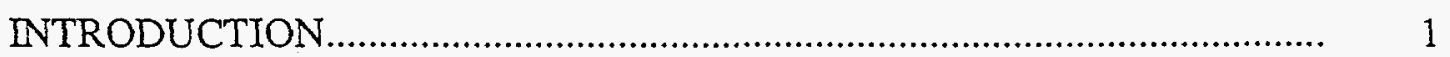

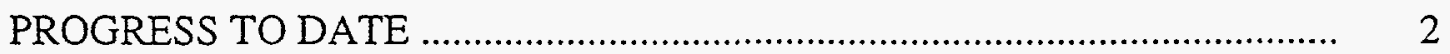

WORK PERFORMED DURING THIS QUARTER......................................... 2

SUMMARY AND CONCLUSIONS.............................................................. 5

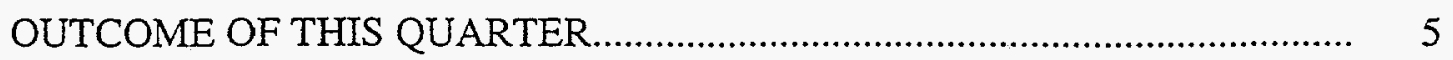

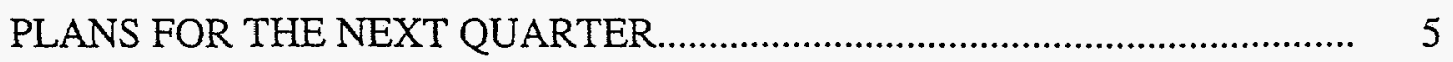

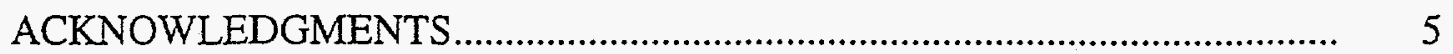

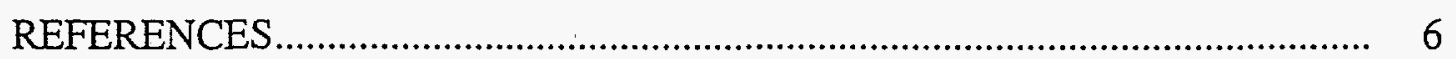

\section{APPENDIX}

Minutes of the Project Review Meeting 


\section{-INTRODUCTION}

This quarterly technical progress report describes work performed under DOE Grant No. DE-FG22-94MT94011 during the period April 01, 1996 to June 30, 1996 which covers the seventh quarter of the project.

Powered infrared (PIR) burners consist of a pressurized air supply, an air/fuel mixing chamber, and a porous metallic or ceramic radiant tile. PIR burner is a surface combustor that elevates the temperature of the burner head to a radiant condition. The principle of the infrared burner is as follows [1]: A premixed fuel/air gas mixture enters a highly porous ceramic or metal layer; the mixture is gradually heated inside the layer and combusted, while it is flowing through the layer. The combustion takes place within the layer. The enthalpy of combustion released in the gas phase heats the porous matrix which then emits thermal radiation from the surface to a heat load.

PIR burners operate with uniform heat transfer, high efficiency, and low $\mathrm{NO}_{\mathrm{x}}$ emissions. Applications for PIR burners include boilers, air heaters, process heaters, immersion heaters, and commercial ovens and fryers. One main reason for the present interest in this type of burner is its low $\mathrm{NO}_{\mathrm{x}}$ emissions. This is attributed to the fact that a large proportion of the heat of combustion is given out as radiation from the burner surface. This results in relatively low gas temperature in the combustion zone compared to that of a conventional free-flame burner. As a consequence, such burners produce less $\mathrm{NO}_{\mathrm{X}}$ mainly by the so called prompt-NO mechanism [2]

The performance of natural gas-fired heating and cooking equipment is strongly dependent on ambient conditions and natural gas composition. In the United States, ambient temperature, pressure, and relative humidity vary significantly by location and season. Also, natural gas compositions supplied by local gas distribution companies exhibit seasonal and regional variations. This is mainly due to the effort for these gas companies to meet the changing demands from users including industrial, commercial and domestic gas applications. To meet the peak demand in the most economic way, it is a common practice to supplement the existing supply with a readily available gas such as liquefied petroleum gas. In service, PIR burners have had reliability and performance problems, especially when exposed to various gas compositions, operating altitudes, and other ambient conditions like temperature and humidity. Fuel gases having different heating values or specific gravities invariably have different chemical compositions. These parameters effect the composition of the gaseous emissions from these burners. Burning characteristics of such gases will differ in important respects, one of the most being speed of flame propagation. Generally, the gas company must make sure that the standby fuel will, when mixed with the base natural gas in the maximum proportion to which it is to be utilized, permit the continued satisfactory functioning of appliances. It is the responsibility of the manufacturers to design appliances capable of performing more satisfactorily under reasonably wide variations in gas composition while retaining desirable efficiencies and operation.

There have been very limited studies to investigate the effects of gas composition upon the performance of radiant burner. Due to the lack of data and fundamental understanding, the 
IR burner product development in the industry is empirical in nature, and is conducted with one gas composition. The objectives of this project are to characterize the operation of IR burner at various gas compositions and ambient conditions and develop a baseline theoretical analysis to predict the behavior of these burners to the change in fuel compositions and ambient conditions.

\section{PROGRESS TO DATE}

This report covers the seventh quarter of the project. In this project, a unique radiation and emission measurement system has been developed at CAU's Combustion Laboratory to study the effects of gas compositions upon the performance of porous radiant burners. It consists of a PIR commercial deep fat fryer that was modified to allow in-situ radiation measurements on the surface of the infrared bumer via a view-port installed through the oil vat. A system 2000 FTIR from Perkin Elmer is used for in-situ measurements of the radiant output from the surface of the burner. A blackbody with a temperature range of 50 to 1200 degree $C$ (model IR-564 from Graseby Infrared) was used to calibrate the FTIR. A set of Horiba gas analyzers are used to measure the emissions from the burner. Experiments were conducted for an extensive test matrix of fuel gas mixtures that represent the complete range of gas compositions usually encountered in the United States. Methane was used as the baseline fuel. Mixtures of methane/propane, methane/hydrogen, and methane/nitrogen along with one propane-air peakshaving gas mixtures were tested to study the effect of fuel mixtures on the performance of the radiant burners. The performance of the burner was investigated for radiant efficiency (ratio of radiative flux escaping the burner to the heat released by combustion), and gaseous emissions at each gas composition and air/fuel ratio.

The above measurements yielded one conference paper, one symposium proceedings paper, and one student poster paper that can be obtained elsewhere [3-5].

A number of published research papers on modeling of porous bumers were reviewed. The physical mechanism and theoretical analysis of the combustion process of the PIR burner were formulated. The numerical modeling and implementation of a PIR burner code at CAU's computing facility is in progress.

\section{WORK PERFORMED DURING THIS QUARTER}

Propane/air (50/50 peakshaving) mixtures were tested and radiation and emission measurements were made for various air flowrates. This completed the testing phase of the project designed in the test matrix submitted in the previous quarterly report. It should be noted that three base limit gases, methane/propane (sooting), methane/hydrogen (lightback), and methane/nitrogen (flame lift), along with one propane-air peakshaving gas mixture were chosen to represent the full range gas supply variation found in the United States. For ranges of distributed gas compositions in US (typical three component mixtures) and in Europe (typical two component mixtures), see Table 1 [6]. The air/fuel ratio in terms of theoretical combustion air (TA) was varied between $90 \%-170 \%$ for each fuel mixture. The baseline TA was set at 
$130 \%$. Analysis of the raw data that started with methane/propane experiments in the previous quarter was continued in this quarter for the rest of the mixtures tested.

\begin{tabular}{|c|c|c|c|c|c|c|c|c|c|}
\hline \multirow[b]{2}{*}{ CONSTITUENTS } & \multicolumn{2}{|c|}{ U.S.NG Variations } & \multicolumn{4}{|c|}{ European Test Gases (2nd Family, Group $\mathrm{H}$ ) } & \multicolumn{3}{|c|}{$\begin{array}{l}\text { Propane-Air Peakshaving (Nomal Winter Range) } \\
\text { (Assume NG }=100 \% \text { Methane for Mixtures) }\end{array}$} \\
\hline & $\begin{array}{l}\text { U.S.NG } \\
\text { Mean }\end{array}$ & $\begin{array}{l}\text { U.S. NG (Colorado) } \\
\text { Min CH4 } \\
\text { Max C2 } 2+6 . \mathrm{C}_{3} \mathrm{H} 8\end{array}$ & $\begin{array}{l}\mathrm{G} 20 \\
\text { Reference }\end{array}$ & $\begin{array}{c}\mathrm{G} 21 \\
\text { Incomplete combust } \\
\text { and sooting limit gas }\end{array}$ & $\begin{array}{c}G 222 \\
\text { Light back } \\
\text { limit gas }\end{array}$ & $\begin{array}{c}\text { G23 } \\
\text { Fame litt } \\
\text { limit gas } \\
\end{array}$ & $\begin{array}{l}\text { Max P-A/NG mix } \\
(60 / 40 \text { P/A mix }) \\
(60 / 40 \text { P-ANG mix })\end{array}$ & $\begin{array}{l}\text { Min P-A/NG mix } \\
(39 / 61 \text { P/A mix }) \\
(10 / 90 \text { P-ANG mix })\end{array}$ & $\begin{array}{l}\text { Avg P-A NG mix } \\
\text { (52/48 P/A mix) } \\
(26.7 \pi 3.3 \text { P-ANG mix) }\end{array}$ \\
\hline Methane (CH4) & $93.70 \%$ & $74.50 \%$ & $100.00 \%$ & $87.00 \%$ & $77.00 \%$ & $92.50 \%$ & $40.00 \%$ & $90.00 \%$ & $73.30 \%$ \\
\hline Ethane $\quad(\mathrm{C} 2 \mathrm{H} 6)$ & $3.20 \%$ & $13.30 \%$ & & & & & & & \\
\hline Propane (C3H8) & $0.70 \%$ & $2.60 \%$ & & $13.00 \%$ & & & $36.00 \%$ & $3.90 \%$ & $13.90 \%$ \\
\hline Hydrogen $\left(H_{2}\right)$ & & & & & $23.00 \%$ & & & & \\
\hline Inerts & $2.40 \%$ & $9.60 \%$ & & & & $7.50 \%$ & $19.00 \%$ & $4.80 \%$ & $10.10 \%$ \\
\hline Oxygen (O2) & & & & & & & $5.00 \%$ & $1.30 \%$ & $2.70 \%$ \\
\hline Total & $100.00 \%$ & $100.00 \%$ & $100.00 \%$ & $100.00 \%$ & $100.00 \%$ & $100.00 \%$ & $100.00 \%$ & $100.00 \%$ & $100.00 \%$ \\
\hline Physical Properties & & & & & & & & & \\
\hline Heating Value, Btu/sef (Gross) & 1023 & 1055 & 1012 & 1209 & 854 & 936 & 1313 & 1000 & 1093 \\
\hline Specitic Gravity & 0.586 & 0.683 & 0.554 & 0.680 & 0.427 & 0.585 & 1.009 & 0.619 & 0.745 \\
\hline Theor AF Ratio (vol) & 9.62 & 9.93 & 9.52 & 11.38 & 7.33 & 8.81 & 12.14 & 9.43 & 10.16 \\
\hline Wobbe No. Btw'sct & 1336 & $12 \pi$ & 1360 & $\$ 466$ & 1308 & 1224 & 1308 & 1283 & 1266 \\
\hline
\end{tabular}

Table 1. Gas Composition Ranges [Ref. 6]

An industrial visit to our industrial partner, Solarnics Inc., MI, a known IR burner manufacturer, was made on July 2, 1996 to discuss and maximize the use of results of this project and better understand burner application issues confronted by infrared power burners. A project review meeting was held at AGAR on the following day in Cleveland, Ohio, to which gas company funders of this project were present. Minutes were distributed under separate cover. An overview of the discussions made in the review meeting is enclosed.

Research collaboration to successfully complete the modeling effort anticipated in this project was made with Energy International (ED) during this quarter. As of July 01, 1996, AGA Research is now a division of EI. Assistance EI can provide in the CAU modeling effort was explored. EI conducted a separate literature review on the radiant burner modeling and concurred with the conclusion of the literature review of CAU made previously; i.e., the most sophisticated model reported in the literature was developed by Sathe et. al. [1] at Arizona State University (ASU). The model developed at ASU incorporates the effects of convection, conduction, radiation, and combustion on the performance of radiant burners. The flow is assumed to be one-dimensional, steady, and laminar. The solid matrix is assumed to be gray and to emit, absorb, and scatter radiant energy. Gaseous radiation is neglected compared to solid radiation. Non-local thermal equilibrium between the gas and the solid phase is accounted for by considering separate energy equations for the two phases.

The PRB model developed and tested by Sathe et al. was based on a modified version of the PREMTX code developed at Sandia National Laboratory in the early 1980's. PREMLX is a 
subset of the CHEMKIN ${ }^{1}$ solution routines, and solves the governing equations for a onedimensional, laminar premixed flame. Sathe et al. added the solid energy conservation equation and the radiative transfer equation to the PREMLX solution algorithm as well as added a term in the PREMIX gas phase energy equation to account for the thermal conduction heat transfer from the gas to the solid burner. Details of the governing equations and solution algorithm can be found in the paper by Sathe et al.

Sathe et al. used a simple single step methane oxidation mechanism for predicting the combustion of methane into carbon dioxide and water. It is unclear from the published results if this simple mechanism was used to reduce computational time, or if fundamental modifications to PREMLX were made which eliminated its capability of incorporating multi-step kinetics. Reasonable agreement between the predicted and measured radiant output, flame speed, and radiant efficiency are reported by the ASU team.

In the current investigation, the model developed by Sathe et al. will be acquired from ASU. A purchase order from CAU has already been received by ASU and currently ASU is putting together the necessary modules to ftp the files to CAU. The model will be reviewed to determine its current capabilities and limitations. It is anticipated that the ASU model will have to be modified to account for variable fuel composition, aeration effects, and operation at altitude. The extent of the modifications will not be known until the ASU model is reviewed, but it is anticipated that coupling the model with the most recent version of CHEMKIN (currently known as CHEMKIN-In) will provide the necessary capabilities for the model to account fuel composition, aeration, and altitude, as well as improve solution convergence. CHEMKIN-II is capable of incorporating pressure sensitive reaction rates, unlike the original version of CHEMKNN, which will be necessary for determining PRB performance at altitude. The updated version of PREMIX also provides improved convergence over the original version used in the ASU code. This improved convergence and stability provided by the updated PREMLX can significantly decrease the computational cost associated with obtaining a converged solution.

Upon the arrival of the ASU code at CAU, model modification and application based on the above discussions will be performed. EI will share the work load with CAU in terms of model modification and application to predict the radiant efficiency measurements made in this project.

During this reporting period, a symposium paper on the effect of natural gas composition variations on porous radiant burners was submitted to the 1996 American Flame Research Committee International Symposium to be held in Baltimore, Maryland, during September 30 October 02, 1996.

\footnotetext{
' CHEMKIN is a software package whose purpose is to facilitate the formation, solution, and interpretation of problems involving elementary gas-phase chemical kinetics. The CHEMKIN library is a collection of about 100 highly modular FORTRAN subroutines that may be called to return information on equation of state, thermodynamic properties, and chemical production rates. The CHEMKIN package is also bundled with solution routines which solve the equations for perfectly stired reactors (PSR), one dimensional laminar flames (PREMIX), or onedimensional shocks (SHOCK).
} 


\section{SUMMARY AND CONCLUSIONS}

A porous radiant burner testing facility consisting of a commercial deep-fat fryer, an FTIR based spectral radiance measurement system, a set of flue gas analysis components, and a fuel gas mixing station was constructed. The measurement capabilities of the system were tested using methane and the test results were found to be consistent with the literature. Following the validation of the measurement system, various gas mixtures were tested to study the effect of gas compositions have on burner performance. Results indicated that the emissions vary with fuel gas composition and air/fuel ratio. The maximum radiant efficiency of the burner was obtained close to air/fuel ratio of 1 .

The project is progressing well and the tasks outlined to this date have been completed on-schedule. During this quarter, experiments for variations in fuel composition were completed. Data analysis and numerical modeling activities to predict the behavior of the burner to the changes in fuel compositions and ambient conditions are aggressively pursued.

\section{OUTCOME OF THIS QUARTER}

A full paper entitled "An Experimental Study of the Effects of Natural Gas Composition Variations on Fan Powered Infrared (PIR) Burners" has been submitted for inclusion in the 1996 American Flame Research Committee (AFRC) International Symposium proceedings, September 30 - October 2, 1996, Baltimore, Maryland.

\section{PLANS FOR THE NEXT QUARTER}

Analysis of the FTIR Raw data and that of the Horiba gas analyzers will be completed to understand the effect of fuel blends on the performance of the burner and its associated behaviors.

Altitude tests will be conducted by AGAR/EI at the Mountain Fuel Supply facility, Utah.

Acquisition, review, and sensitivity analyses of the key parameters of the ASU code to modify and predict the burner behavior to the change in fuel compositions will be conducted.

\section{ACKNOWLEDGMENTS}

This project is supported by PETC/DOE through Contract No. DE-FG22-94MT94011 and by Atlanta Gas Light Company, Brooklyn Union Gas Company, Columbia Gas Distribution Companies, National Fuel Gas Distribution Corporation. Technical discussion on the burner application issues provided by Solarnics, Inc. is appreciated. 


\section{REFERENCES}

1. Sathe, S. B., Kulkarni, M. R., Peck, R. E., and Tong, T. W., Twenty-Third Symposium (International) on Combustion/The Combustion Institute, 1990/pp. 1011-1018.

2. Williams, A., Woolley, R., and Lawes, M., The Formation of $\mathrm{NO}_{\mathrm{x}}$ in Surface Burners, Combustion and Flame 89: 157-166 (1992).

3. Bai, T. J., Yeboah, Y. D., and Sampath, R., Effects of Gas Composition upon the Performance of Infrared Radiant Burners, Fourth Annual HBCU conference organized by PETC/DOE at North Carolina A \& T, April 2-4, 1996.

4. Bai, T. J., Yeboah, Y. Y., Sampath, R., Farnsworth, C., and Mocsari, J., An Experimental Study of the Effects of Natural Gas Composition Variations on Fan Powered Infrared (PIR) Burners, 1996 American Flame Research Committee (AFRC) International Symposium, September 30 - October 2, 1996, Baltimore, Maryland.

5. Arthur, A., James, L., Wang, Z., Bai, T. J., Yeboah, Y. D., and Sampath, R., A Study of Infrared Energy and Gaseous Emissions Generated by Porous Radiant Burners, poster paper at the Fourth Annual HBCU conference organized by PETC/DOE at North Carolina A \& T, April 2-4, 1996.

6. Liss, W. E., Variability of Natural Gas Composition in Select Major Metropolitan Areas of the United States, GRI - 92/0123, March 1992. 


\title{
CAU/AGAR IR Burner Project \\ Minutes of July 2,1996 \\ Project Review Meeting \\ AGA Research
}

\author{
Attendees: \\ Dr. Teijun Bai, CAU \\ Dr. Ramamthan Sampath, CAU \\ Mr. Vinton Wolfe, Atlanta Gas Light \\ Mr. Steve Petty, Cohumbia Gas \\ Mr, Clifford Andrews \\ Mr. Farshid Ahmady, Solaronics \\ Mr. Craig Farnsworth, AGAR
}

\section{Meeting Notes:}

It was noted that $C A U$ and $A G A R$ personnel met at Solaronics on the previous day to carry forward discussions regarding means to maximize usefulness of results of this project and better understand burner application issues confronted by infared power burners.

It was also noted that as of July 1, AGA Research is now a division of Energy International (EI). Information regarding EI is Attachment \#1.

Meeting presentation is Attachment $\# 2$.

An overview of the discussion is as follows:

- The gas composition and burner aeration tests are $95 \%$ completed. It was noted that ethane was not included in test matrix. It was observed by LDC personnel that this is a significant secondary component in much of the natural gas distributed in the US and should be addressed in this program.

- The development of the model has been hampered by inability to obtain desired combustion kinetics code from Arizona State University.

- Given the non-availability of proposed barometric environmental chamber at AGAR it was proposed that a series of tests be performed at AGAR with variation of temperature and humidity and at Mountain Fuel Supply at various altitudes. The proposed chamber was to be capable of varying barometric pressure (altitude), temperature; and humidity. It was decided that only the altitude testing was directly pertinent to the subject project. Mountain Fuel Supply has agreed to make their mobile laboratory available to this project at no cost.

- It was decided that maximum effort should be placed on making sure that the computer model results predict the measured results of experiments as closely as possible.

- Additional analytical and modeling capabilities have been added to the AGAR staff since the inception of this project. It was agreed that assistance from AGAR in the modeling effort would be explored. (Subsequent to the meeting it was found that EI has been heavily involved in the area of combustion modeling. EI staff have supplied copy of kinetics code noted above and discussions are underway to determine appropriate assistance that can be provided to CAU by EI.) At the completion of these discussions, revised scope of work for AGAR effort will be submitted for approval. 
- Appropriate follow-on activity to the current project was discussed. It was agreed that, given suitable agreement of modelod and experimental results with the model currently under development, the following additions would be beneficial:

- Add ethane as secondary component (currently proparte, nitrogen, hydrogen, and propane-air are considered)

- Consider multi-component mixtures beyond current binary (plus air) mixtures

- Make model 2-dimensional

- Add burner/heat exchanger environment characteristics (1.e., proximity of heat exchanger surface, radiant scattering, back pressure, radiant heat sink, downstrearn flow field, etc.)

- Surface characteristics of burner (generalize beyond tile burner)

- Bumer shape

- Combustion air humidity

Paper presented by CAU at HBCU conference in April is attachment \#3. Abstract of paper to be presented at American Flame Research Conference is attachment \#4.

\section{The following is a summary of action item identified at this meeting:}

- Explore assistance AGAR/EI can provide in the CAU modeling effort. (R. Sampath and C. Farnsworth)

- Submit revised scope of AGAR activity. (C. Famsworth)

- Complete gas composition and bumer aeration experiments. (R. Sampath)

- Carry out testing at Mountain Fuel Supply. (C. Farnsworth)

\section{DISCLAIMER}

This report was prepared as an account of work sponsored by an agency of the United States This rester Government nor any agency thereof, nor any of their Government. Neither the United States Government nor any agency thegal liability or responsibility for the accuracy, completeness, or usefulness of any information, apparatus, product, or brocess disclosed, or represents that its use would not infringe privately owned rights. Reference herein to any specific commercial product; process, or service by trade name, trademark, manufacturer, or otherwise does not necessarily constitute or imply its endorsement, recommateres mendation, or favoring by the United States Government or any agency the reflect those of the United States Government or any agency thereof. 